\title{
Phosphatase and tensin homolog overexpression decreases proliferation and invasion and increases apoptosis in oral squamous cell carcinoma cells
}

\author{
QI GAO $^{1}$, LEI ZHANG ${ }^{1}$, BIN ZHANG $^{2}$, QI-YU WANG ${ }^{2}$, CHANG-FU SUN $^{3}$, XIAO-TING DONG ${ }^{1}$ and JANG YING ${ }^{2}$ \\ ${ }^{1}$ Department of Biochemistry, Liaoning Medical University; ${ }^{2}$ Department of Stomatology, \\ The First Affiliated Hospital of Liaoning Medical University, Jinzhou, Liaoning 121001; \\ ${ }^{3}$ Department of Oral and Maxillofacial-Head and Neck Surgery, School of Stomatology, \\ China Medical University, Shenyang, Liaoning 110002, P.R. China
}

Received December 6, 2013; Accepted May 13, 2014

DOI: $10.3892 / \mathrm{ol} .2014 .2283$

\begin{abstract}
Phosphatase and tensin homolog (PTEN) is a potent tumor suppressor which regulates various cellular functions. The aim of the present study was to analyze the function of PTEN gene expression in squamous cell carcinoma (SCC) cells. This gene exhibits a unique function in cell migration and proliferation during the early stages of embryonic development. However, its role as a tumor suppressor gene in tongue squamous carcinoma cells remains unclear. In the present study, an SCC-4 cell line stably expressing PTEN was established and the effects of PTEN gene expression on SCC-4 cell proliferation, invasion and apoptosis were investigated. PTEN expression was found to induce apoptosis in SCC-4 cells, possibly via negative regulation of the phosphatidylinositide 3-kinase/Akt signaling pathway and increased expression of Bcl-2-interacting mediator of cell death. In addition, PTEN was found to control the epithelial-mesenchymal transition in SCC cells, thereby reducing their invasive ability. Furthermore, Transwell assay revealed that the expression of E-cadherin was increased, while the expression of vimentin and SNAIL was decreased. This study has provided an important insight into the mechanisms by which PTEN mediates the progression and early metastasis of tongue carcinoma.
\end{abstract}

\section{Introduction}

Oral tongue squamous cell carcinoma (OTSCC) is one of the most common types of malignant tumors of the oral and

Correspondence to: Professor Bin Zhang, Department of Stomatology, The First Affiliated Hospital of Liaoning Medical University, No. 2, Section 5, People Street, Jinzhou, Liaoning 121001, P.R. China

E-mail: zhangbinkq@163.com

Key words: phosphatase and tensin homolog, invasion, apoptosis, cell proliferation, SCC-4 maxillofacial region, comprising $32.3 \%$ of all cases of oral cancer. Although the pathogenesis of OTSCC remains unclear, it has been suggested that it may involve the mutation and abnormal expression of multiple genes (1). The prognosis for patients with OTSCC is relatively poor and the risk of relapse is high, which may be attributable to the highly invasive nature of OTSCC cells, the frequent movements of the tongue and the rich blood supply to the tongue. Therefore, early lymph node and late distant metastases are extremely common in tongue cancer.

With recent advancements in molecular biology, molecular genetics and related disciplines, study regarding potential treatments for OTSCC has focused on gene therapy (2). The first tumor suppressor gene with phosphatase activity identified in humans was the phosphatase and tensin homolog (PTEN) gene. Studies have shown that the PTEN gene undergoes significant mutations and deletions in a variety of tumors, including melanoma, breast, prostate and endometrial cancer, resulting in a loss of protein expression or dysfunction, thereby contributing to tumor development $(3,4)$. Additional study has indicated that mutations and deletions in the PTEN gene also promote the growth and development of gliomas and head and neck cancers (5).

The epithelial-mesenchymal transition (EMT) refers to the process whereby skin-derived precursor cells undergo phenotypic changes during the embryonic and tumor progression stages. E-cadherin, a $120-\mathrm{kDa}$ transmembrane glycoprotein, interacts with $\alpha$-, $\beta$ - and $\gamma$-catenins, as well as the E-cadherin/catenin complex, to then associate with the actin microfilament system of the cell, regulating tissue and morphological changes. Thus, the expression and functional status of the E-cadherin/catenin complex within the tumor influences cell separation and adhesion, mediating tumor invasion (6). Vimentin is often considered a marker for tumors of mesenchymal origin, and vimentin expression is increased in numerous epithelial tumors and is closely associated with tumor invasion. SNAIL is a zinc finger protein that binds to the promoter of the E-cadherin gene, inducing tumor cell EMT (7). Vimentin, SNAIL and E-cadherin are closely associated with EMT and may be useful indicators of EMT. Recent study has examined 
the ability of EMT to induce tumor invasion and metastasis (8); however, thus far, no reports have investigated the impact of the PTEN tumor suppressor gene on EMT in OTvSCC (9).

In the present study, PTEN was overexpressed in SCC-4 cells, and the effects of PTEN expression on the proliferation and apoptosis of OTSCC cells was examined. In addition, the correlation between the invasiveness of PTEN-transfected OTSCC cells and EMT-associated markers was investigated.

\section{Materials and methods}

Reagents and antibodies. SCC- 4 cells were provided by the Ninth People's Hospital of Shanghai Jiaotong University (Shanghai, China) and originally purchased from the Cell Bank of the Chinese Academy of Sciences (Shanghai, China). Mouse anti-human vimentin polyclonal antibodies were purchased from Santa Cruz Biotechnology, Inc. (Santa Cruz, CA, USA). Mouse anti-human E-cadherin monoclonal antibodies, rabbit anti-human Akt polyclonal antibodies, rabbit anti-phospho-Akt polyclonal antibodies and rabbit anti-human Bcl-2-interacting mediator of cell death (BIM) polyclonal antibodies were obtained from Jiamay Biotech (Beijing, China). Primer synthesis and DNA sequencing were performed by Wuhan Ying Qi Biotechnology Co., Ltd. (Wuhan, China), MTT and dimethyl sulfoxide were purchased from Promega Corporation (Madison, WI, USA), and tetramethylethylenediamine and sodium dodecyl sulfate were purchased from Sigma-Aldrich (St. Louis, MO, USA).

Cell culture. The OTSCC SCC-4 cell line was maintained at $37^{\circ} \mathrm{C}$ in a humidified incubator with an atmosphere of $5 \%$ $\mathrm{CO}_{2}$ in Dulbecco's modified Eagle's medium (DMEM)/F12 medium (Hyclone; Thermo Fisher Scientific, Rockford, IL, USA) supplemented with $10 \%$ fetal bovine serum (Thermo Fisher Scientific) without any antibiotics.

Human tissue specimens. A total of 40 human tissue specimens were collected from individuals who underwent surgery at the Department of Surgery, First Affiliated Hospital of Liaoning Medical University (Jinzhou, China) between January 2007 and December 2010. Clinical information is summarized in Table I. All patients provided written informed consent and were assessed for PTEN expression. This study was approved by the human ethics committee of Liaoning Medical University.

Immunohistochemistry. Immunohistochemistry was performed using a Streptavidin-Biotin Complex (SABC) kit (Wuhan Boster Biological Technology, Ltd., Wuhan, China) according to the manufacturer's instructions. Briefly, the tissue sections were deparaffinized in xylene for $20 \mathrm{~min}$ and then dehydrated in graded alcohol solutions, followed by detection using the avidin-biotin complex method by SABC kit. The endogenous peroxidase activity was blocked by immersing the sections in $3 \% \mathrm{H}_{2} \mathrm{O}_{2}$ in methanol for $30 \mathrm{~min}$. For antigen retrieval, the sections were heated in $0.01 \mathrm{M}$ citrate buffer $(\mathrm{pH}$ 6.0; Shanghai Xin Biological Technology Co., Ltd., Shanghai, China) for $15 \mathrm{~min}$. The sections were then treated with $10 \%$ normal rabbit serum for $30 \mathrm{~min}$, followed by incubation with mouse anti-human PTEN monoclonal antibodies [1:100 dilution; Santa Cruz Biotechnology (Shanghai) Co., Ltd., Shanghai,China] at
Table I. Clinicopathological features of OTSCC.

Clinicomorphological parameters

$\begin{array}{lr}\text { Age, years } & \\ <20 & 6(15.0) \\ 20-40 & 14(35.0) \\ >40 & 20(50.0) \\ \text { Gender } & \\ \text { Female } & 26(65.0) \\ \text { Male } & 14(35.0) \\ \text { Normal oral tissues }{ }^{\mathrm{a}} & \\ \text { Female } & 10(66.7) \\ \text { Male } & 5(33.4) \\ \text { OTSCC tissues } & 18(72.0) \\ \text { Female } & 7(28.0) \\ \text { Male } & \end{array}$

${ }^{\mathrm{a}}$ Obtained from healthy volunteers. OTSCC, oral tongue squamous cell carcinoma.

$4^{\circ} \mathrm{C}$ overnight. Following incubation with a biotin-conjugated secondary antibody, incubation was performed with streptavidin solution at $37^{\circ} \mathrm{C}$ for $20 \mathrm{~min}$, followed by incubation with $\mathrm{SABC}$ reagents at $37^{\circ} \mathrm{C}$ for $30 \mathrm{~min}$. The tissues were stained with 3,3'-diaminobenzidine (Chinese sales platform ELISA kits, Shanghai, China). Negative and positive controls were conducted in each run of immunohistochemistry. A total of five to six fields from each tissue section was selection, and 100 cells from each field were counted (Countstar automated cell counter, Biomen Biosystems Co., Ltd., Guangzhou, China) at a final magnification of x400 (Olympus BX43; Shanghai Zeshi Photoelectric Technology Co., Ltd., Shanghai, China). The evaluation was performed by two independent pathologists, without any prior knowledge of each patient's clinical information (Fig. 1).

Expression of PTEN $m$ RNA by reverse transcription-polymerase chain reaction $(R T-P C R)$. Total RNA was extracted from SCC-4 cells using the TRIzol method (Gibco-BRL, Carlsbad, CA, USA). PTEN total RNA was amplified using RT-PCR. The amplification system and conditions were based on the manufacturer's instructions stated in the Takara One-Step RNA PCR kit (Takara Bio, Inc., Shiga, Japan). Primer Premier 5.0 software (PREMIER Biosoft, Palo Alto, CA ,USA) was used to design primers for the PTEN gene based on sequences retrieved from GenBank. The upstream and downstream primers were 5'-GCCGAATTCGACTTTTGTAATTTGTGTA-3' and 5'-CCGCTCGAGCAGTCGCTGCAACCATCCA-3', respectively, with EcoRI restriction sites introduced to the 5' ends for nucleotide protection. For RT-PCR, each reaction was carried out as follows: Denaturation at $94^{\circ} \mathrm{C}$ for $5 \mathrm{~min}$; 60 cycles of $94^{\circ} \mathrm{C}$ for $60 \mathrm{sec}, 60^{\circ} \mathrm{C}$ for $60 \mathrm{sec}$ and $72^{\circ} \mathrm{C}$ for $1.5 \mathrm{~min}$; and extension at $72^{\circ} \mathrm{C}$ for $10 \mathrm{~min}$.

Transfection with the PTEN eukaryotic expression plasmids. SCC-4 cells growing at the logarithmic growth phase were seeded onto six-well plates and transfections were performed 

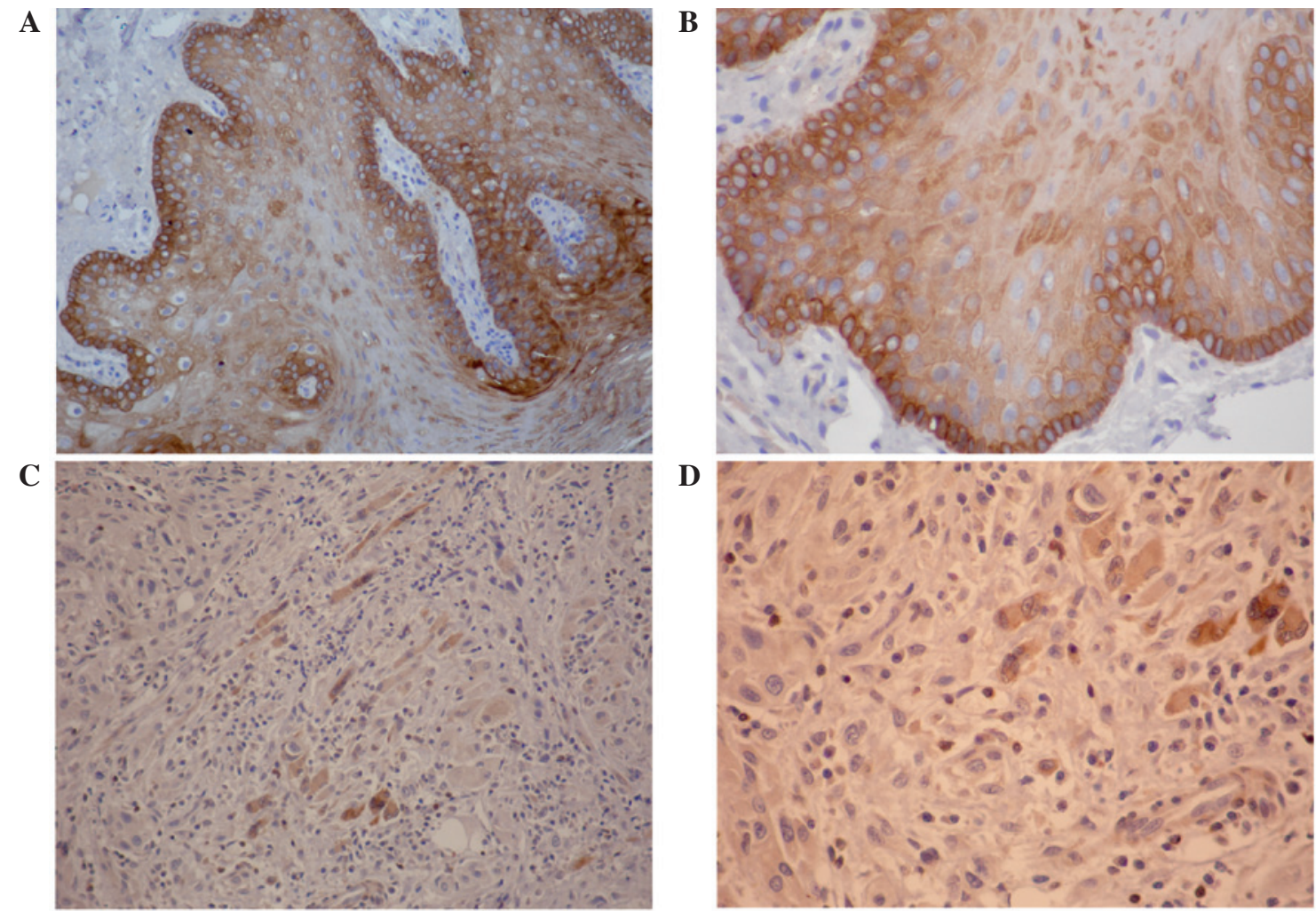

Figure 1. Immunohistochemical staining of PTEN in normal oral tissue and OTSCC tissue. Cytoplasmic expression of PTEN in normal oral tissue at magnifications of (A) x200 and (B) x400. Loss of PTEN expression in OTSCC tissue at magnifications of (C) x200 and (D) x400. PTEN, phosphatase and tensin homolog; OTSCC, oral tongue squamous cell carcinoma.

when the cells reached $70-80 \%$ confluence, using the Lipofectamine 2000 reagent kit (Invitrogen Life Technologies, Carlsbad, CA, USA) according to the manufacturer's instructions. Experiments were carried out using three groups of cells: Cells transfected with phosphorylated (p)-enhanced green fluorescent protein (EGFP)-PTEN recombinant plasmid; cells transfected with pEGFP-N1 empty plasmid; and untransfected control cells. The intracellular expression of GFP was observed under a fluorescence microscope (Olympus BX43) at 24, 48 and $72 \mathrm{~h}$ following transfection. At $48 \mathrm{~h}$ following transfection, the cells were also cultured in DMEM selection medium containing $800 \mu \mathrm{g} / \mathrm{ml} \mathrm{G} 418$. Cloned SCC-4 cells exhibiting stable expression of PTEN were then filtered for amplification and culture, and stable cell lines in the logarithmic growth phase were used for follow-up tests.

Western blotting. Following transfection, cells were subjected to total protein extraction. Protein content was measured against bovine serum albumin, which was used as the standard. Proteins were separated by polyacrylamide gel electrophoresis on $10 \%$ gels, transferred to polyvinylidene fluoride membranes and blocked for $1 \mathrm{~h}$ in $5 \%$ skimmed milk. Following washing with Tris-buffered saline and Tween 20 , membranes were incubated with the primary antibodies (Akt, 1:1,000; phospho-Akt, 1:1,000; BIM, 1:1,000; and PTEN, 1:500) and then incubated overnight at $4^{\circ} \mathrm{C}$ (PTEN) or room temperature (Akt, phospho-Akt and BIM). Next, the membranes were washed and incubated with a sheep anti-mouse polyclonal horseradish peroxidase-conjugated secondary antibody (Jiamay Biotech) for $1-2 \mathrm{~h}$ at room temperature. The Biospectrum imaging system (Beijing Dequan
Development Trading Co., Ltd., Beijing, China) was used for image capture. The optical density of each band was measured using ImageJ software (National Institutes of Health).

Cell proliferation assays. Following transfection, cells at the logarithmic growth phase were used for cell proliferation assays. The cell concentration was adjusted to $1 \times 10^{4}$ cells $/ \mathrm{ml}$, and $100 \mu \mathrm{l}$ cell suspension was added to each well of a 96-well plate ( $n=42$ wells/cell group). Every day between day one and seven, cells were counted to measure proliferation and MTT assays were performed according to standard instructions to confirm cell viability ( $\mathrm{n}=3$ wells/day per cell group for each of the proliferation and MTT assays).

Statistical analysis. All statistical analyses were performed using SPSS software, version 13.0 (SPSS, Inc., Chicago, IL, USA). Differences between all three groups were determined using analysis of variance tests, while differences between two groups were analyzed by Student's t-test. $\mathrm{P}<0.05$ was considered to indicate a statistically significant difference.

\section{Results}

Loss of PTEN protein expression in OTSCC. The expression of PTEN protein in OTSCC was primarily cytoplasmic, with infrequent nuclear localization (Fig. 1). PTEN expression was observed in 15 out of 15 (100\%) normal oral tissues; however, loss of PTEN expression was apparent in all 25 OTSSC specimens (Fig. 1). When comparing the expression of PTEN in OTSCC with normal oral tissue, the frequency of PTEN loss 
A

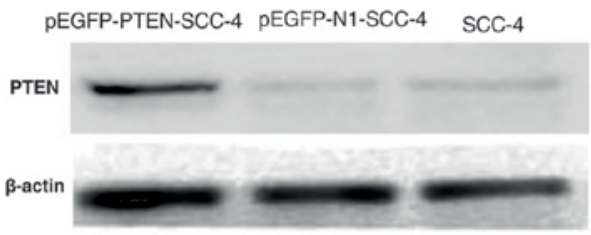

B

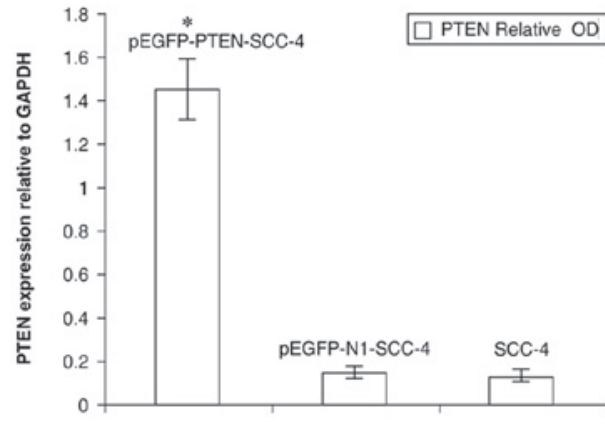

C

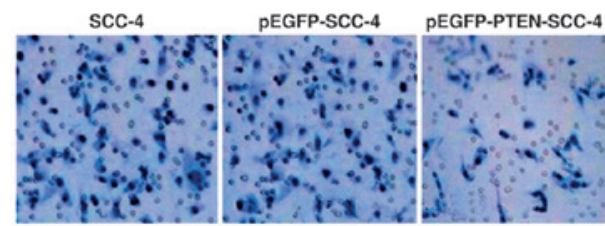

D

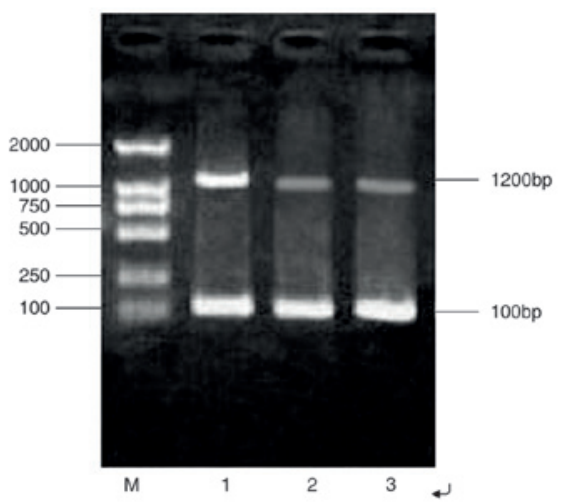

Figure 2. Effects of PTEN vector transfection on PTEN expression in SCC-4 cells. (A) Untransfected cells (lane 1) and cells transfected with empty vector (lane 2) or PTEN (lane 3) were subjected to western blotting using anti-PTEN antibodies. $\beta$-Actin was used as a loading control. (B) Quantification of western blots to measure PTEN protein expression. The OD of PTEN (relative to GAPDH) was as follows: $1.45 \pm 0.14,0.17 \pm 0.02$ and $0.15 \pm 0.03$ in the pEGFP-PTEN-SCC-4, pEGFP-N1-SCC-4 and SCC-4 groups, respectively. (C) Effects of PTEN expression on the invasion of SCC-4 cells. Untransfected SCC- 4 cells and cells transfected with pEGFP-SCC-4 or pEGPF-PTEN-SCC-4 were subjected to Transwell invasion assays. The number of cells with penetrated membranes in the pEGPF-PTEN-SCC-4 group was evidently less than in the SCC-4 and pEGFP-SCC-4 groups. (D) Reverse transcription-polymerase chain reaction for PTEN gene mRNA expression. M, DNA maker; 1, pEGFP-PTEN-SCC-4; 2, pEGFP-N1-SCC-4; and 3, SCC-4. PTEN, phosphatase and tensin homolog; pEGFP, phosphorylated enhanced green fluorescent protein; SCC, squamous cell carcinoma; OD, optical density.

was statistically significant $(\mathrm{P}<0.001)$. Notably, the expression of PTEN was not associated with age, gender or histological grade (data not shown).

PTEN mRNA expression levels in each cell group. The results of $1 \%$ agarose gel electrophoresis revealed that the three groups of cells appeared in $~ 1,200$-bp bands. However, the pEGFP-PTEN-SCC-4 group was found to exhibit significantly thicker bands (Fig. 2D).

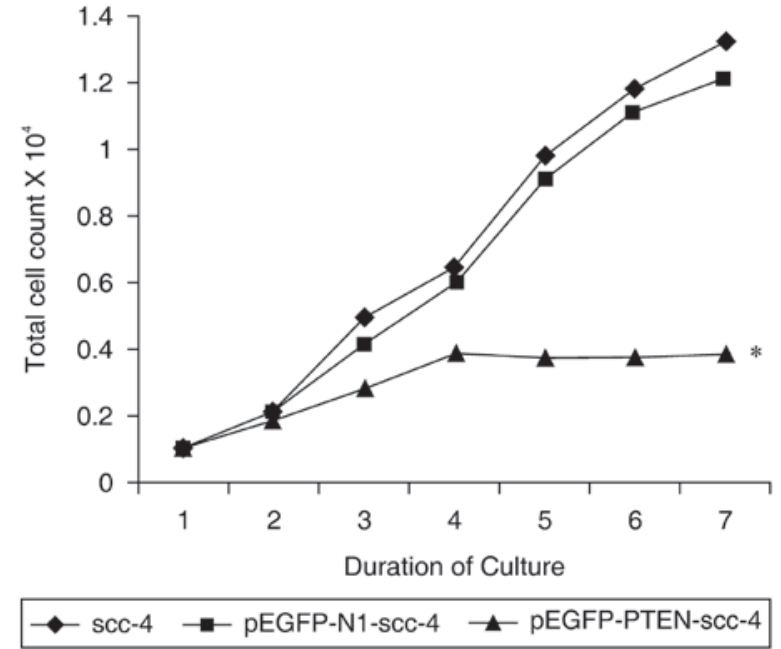

Figure 3. PTEN expression suppresses SCC-4 cell proliferation. Cell proliferation was measured by counting the number of cells each day for seven days PTEN, phosphatase and tensin homolog; SCC, squamous cell carcinoma, pEGFP, phosphorylated enhanced green fluorescent protein.

PTEN expression in SCC-4 cells. Western blotting results revealed that the transfected group exhibited a significantly increased brightness of bands when compared with the other groups. The optical density of PTEN protein expression (relative to GAPDH) in PTEN-transfected cells was $1.07 \pm 0.15$, which was identified to be significantly different when compared with that of cells transfected with the empty vector and untransfected cells $(0.62 \pm 0.11$ and $0.57 \pm 0.08$, respectively; $\mathrm{P}<0.05$; Fig. $2 \mathrm{~A}$ and $\mathrm{B})$. These results indicated that transfection with the PTEN-containing plasmid induced overexpression of exogenous PTEN.

PTEN overexpression suppresses SCC-4 cell proliferation. The effects of PTEN overexpression on SCC-4 cell proliferation were investigated. Notably, transfection with the pEGFP-PTEN-SCC-4 vector resulted in a significant reduction in cell proliferation when compared with that of the pEGFP-N1-SCC-4 and SCC-4 groups following the third day of culture $(\mathrm{P}<0.01$; Fig. 3). These results indicated that PTEN expression suppresses SCC-4 cell proliferation.

Overexpression of PTEN induces apoptosis in SCC-4 cells. Flow cytometry analysis of Annexin V-phycoerythrin-Cy5/propidium iodide double-staining indicated that the percentage of apoptotic cells was significantly higher in PTEN-GFP-transfected cells when compared with that of cells transfected with the empty vector and untransfected control cells $(48.1 \pm 2.6,1.2 \pm 0.7$ and $1.4 \pm 0.9 \%$, respectively) $48 \mathrm{~h}$ following transfection. No significant differences in apoptotic rate between the control and empty vector groups were identified $(\mathrm{t}=0.3 ; \mathrm{P}>0.05)$. However, a significant difference was identified between the apoptotic rate of the experimental group and that of the other two groups $(\mathrm{t} 1=30.2 ; \mathrm{t} 2=29.4 ; \mathrm{P}<0.01)$ (Fig. 4).

Effects of PTEN expression on Akt, phospho-Akt and BIM levels in tongue cancer. No significant differences in total Akt expression were identified among transfected and 

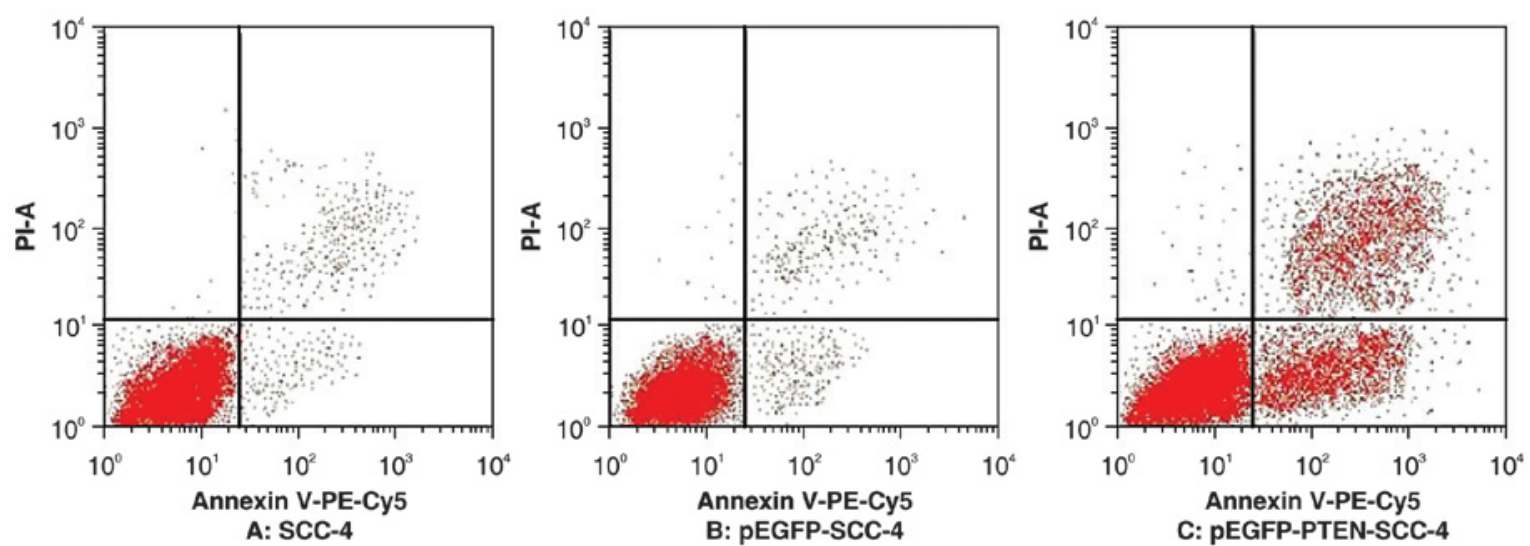

Figure 4. Analysis of apoptosis in SCC-4 cells by flow cytometry. At $48 \mathrm{~h}$ following transfection, cells were stained with Annexin V-PE-Cy5/PI and subjected to flow cytometry analysis. SCC, squamous cell carcinoma; PE, phycoerythrin; PI, propidium iodide; pEGFP, phosphorylated green fluorescent protein; PTEN, phosphatase and tensin homolog.

A

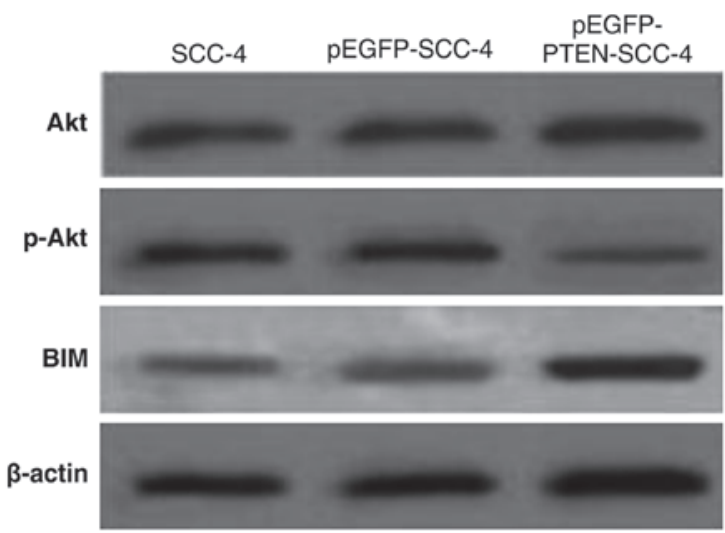

B

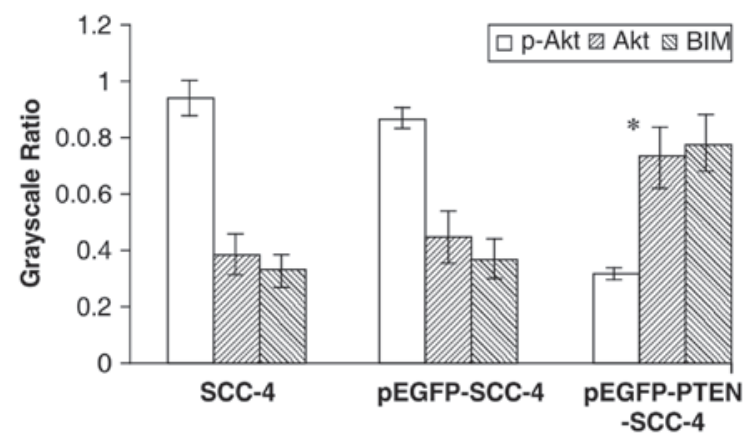

Figure 5. (A) Effects of PTEN expression on Akt, phospho-Akt and BIM levels in SCC-4 cells. Untransfected cells (lane 1) and cells transfected with empty vector (lane 2) or PTEN (lane 3) were subjected to western blotting using the indicated antibodies. $\beta$-actin was used as a loading control. (B) Quantification of phospho-Akt, Akt and BIM protein expression. PTEN phosphatase and tensin homolog; BIM, Bcl-2-interacting mediator of cell death; pEGFP, phosphorylated enhanced green fluorescent protein; SCC, squamous cell carcinoma.

untransfected cells following western blotting. However, while phospho-Akt levels were $0.94 \pm 0.13$ for SCC- $4,0.87 \pm 0.04$ for pEGFP-SCC- 4 and $0.32 \pm 0.02$ for pEGFP-PTEN-SCC- 4 $(\mathrm{P}>0.05)$, while phospho-Akt levels were significantly reduced in PTEN-transfected cells $(\mathrm{P}<0.05)$. The expression of BIM, a pro-apoptotic, BH3-only protein member of the Bcl-2 family which is critical in apoptosis (10), was $0.33 \pm 0.06$ for SCC-4, $0.37 \pm 0.07$ for pEGFP-SCC-4 and $0.78 \pm 0.10$ for
A

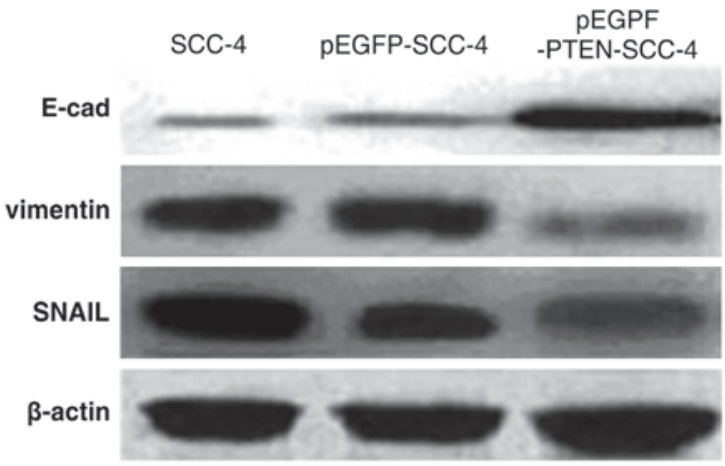

B

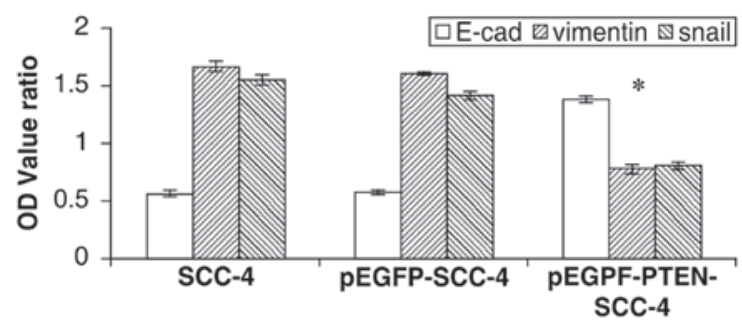

Figure 6. (A) Effects of PTEN expression on E-cadherin, SNAIL and vimentin proteins levels in SCC-4 cells. Untransfected cells (lane 1) and cells transfected with empty vector (lane 2) or PTEN (lane 3) were subjected to western blotting using the indicated antibodies. $\beta$-Actin was used as a loading control. (B) Quantification of E-cadherin, SNAIL and vimentin protein expression. PTEN, phosphatase and tensin homolog; SCC, squamous cell carcinoma; pEGFP, phosphorylated enhanced green fluorescent protein; OD, optical density.

pEGFP-PTEN-SCC-4; however, it was found to be significantly upregulated in PTEN-transfected cells $(\mathrm{P}<0.01)$ (Fig. 5).

Overexpression of PTEN inhibits SCC-4 cell invasion. Cells in the control group (SCC-4), empty vector group (pEGFP-SCC-4) and transfected group (pEGFP-PTEN-SCC-4) were cultured in Transwell invasion chambers for $36 \mathrm{~h}$. For the 30 visually selected fields from each group, the numbers of cells invading through the membrane were $82 \pm 5,80 \pm 4$ and $42 \pm 5$, respectively (Fig. 2C). These results demonstrated that PTEN expression caused a significant reduction in SCC-4 invasion when compared with the control $(\mathrm{P}<0.01)$. 
In addition, a statistically significant difference was identified when comparing the expression of the three proteins in the transfected and control groups $(\mathrm{P}<0.05)$. However, no significant differences were identified between the protein expression of the empty vector and control groups $(\mathrm{P}>0.05)$. The results of the western blotting revealed that PTEN expression significantly induced the expression of E-cadherin (SCC-4, 0.556 \pm 0.022 ; pEGFP-SCC-4, 0.573 \pm 0.013 ; and pEGFP-PTEN-SCC-4, 1.375 \pm 0.026$)$ and suppressed the expression of SNAIL (SCC-4, 1.554 \pm 0.041 ; pEGFP-SCC-4, 1.412 \pm 0.036 ; and pEGFP-PTEN-SCC-4, 0.801 \pm 0.027$)$ and vimentin (SCC-4, 1.667 \pm 0.045 ; pEGFP-SCC-4, 1.593 \pm 0.013 ; and pEGFP-PTEN-SCC-4, 0.778 \pm 0.032$)(\mathrm{P}<0.01)$ (Fig. 6). These results suggested that PTEN may block OTSCC cell invasion by inhibiting the EMT process.

\section{Discussion}

PTEN is a tumor suppressor gene with dual phosphatase activity. However, its mechanism of action is not fully understood (11). At present, PTEN is considered to convert dephosphorylated phosphatidylinositol $(3,4,5)$-triphosphate $\left(\mathrm{PIP}_{3}\right)$ to phosphtidylinositol $(4,5)$-bisphosphate, thereby blocking $\mathrm{PIP}_{3}$-mediated activation of protein kinase $\mathrm{B} / \mathrm{Akt}$ and suppressing the growth and development of tumors (12). In addition, PTEN has been shown to function in the nucleus and thus may be important in transcriptional regulation, however, its nuclear targets remain unclear (13).

In the present study, PTEN expression was detected in OTSCC specimens and the effects of PTEN expression in SCC-4 cells transfected with a PTEN expression vector were investigated. Using this model, PTEN expression was found to exert a tumor suppressor function, which significantly reduced the proliferation capacity of SCC-4 cells, thus confirming the function of PTEN in the malignant behavior of OTSCC.

Deletion of the BIM gene may lead to tumorigenesis (14). Previous studies have shown that numerous anticancer drugs, including those for lung (15) and ovarian (12) cancer, induce tumor cell apoptosis via the increased expression of BIM. In the current study, the results of the western blotting indicated that BIM expression increased following transfection with a PTEN expression vector in SCC-4 cells, suggesting that PTEN expression may affect the phosphatidylinositide 3-kinase (PI3K)/Akt signaling pathways via upregulation of BIM. The results of this study also confirmed that it is possible to induce apoptosis of SCC-4 cells via in vitro PTEN transfection, possibly through negative regulation of the $\mathrm{PI} 3 \mathrm{~K} / \mathrm{Akt}$ signaling pathway and increased expression of the transcription factor Akt and the pro-apoptotic protein BIM, thereby enhancing SCC-4 cell apoptosis. Since the PI3K/Akt pathway involves a variety of additional factors, further targets for gene therapy are available.

It has been demonstrated that PTEN suppresses tumor development by promoting apoptosis of tumor cells and regulating the cell cycle, reducing the invasiveness of tumor cells in esophageal cancer and melanoma (16). Similarly, in the current study, PTEN expression was reduced in advanced tumors and tumors undergoing lymph node metastasis.

Loss of E-cadherin and upregulation of vimentin are hallmarks of the EMT. In addition, E-cadherin low/vimentin may be used as an indicator of tumor prognosis, whereby a small ratio indicates poor prognosis (18). Furthermore, the transcription factor SNAIL is important in EMT. Häyry et al (19) revealed that in 73 cases of OTSCC, SNAIL expression and depth of invasion were found to significantly correlate, demonstrating that SNAIL directly affects tumor invasion and metastasis. Thus, the differential expression of these three proteins in OTSCC cells indicates the varying degrees of EMT. In addition to the results of the current study regarding cell invasion, these results show that PTEN regulates the expression of E-cadherin vimentin and SNAIL, indicating the involvement of PTEN in EMT. Thus, when considering the invasive abilities of the different groups of cells, we hypothesize that the invasive ability of OTSCC cells is associated with the EMT process.

PTEN gene deletion induces EMT via the PI3K/Akt signaling pathway (20), thereby increasing the invasive ability of tumor cells. Additionally, Leslie et al (21) reported that colorectal cancer cells become spindle-shaped following treatment with LY294002, a PI3K/Akt-specific inhibitor, an effect which was accompanied by a reduced expression of E-cadherin and increased invasiveness of the cells, further supporting the role of the PTEN/E-cadherin signaling axis in EMT.

In conclusion, the PTEN gene is closely associated with the development of SCC. Expression of the PTEN gene may inhibit the growth of tongue SCC cells. This may present a possible line of gene therapy. However, OTSCC is a solid tumor and its structure and biological characteristics are extremely complex. Therefore, the SCC-4 cell line does not fully reflect the tumor itself and cannot present the complete genetic characteristics of OTSCC. Thus, PTEN must be studied using animal models to elucidate its detailed mechanism of action.

\section{Acknowledgements}

This study was supported by The First Affiliated Hospital of Liaoning Medical University (Jinzhou, China), and the generous donations of cancer patients and their families. The authors would like to thank Professor Shu Lin Gao and Professor Qin Yu for valuable support, and Editage/Cactus Communications Pvt. Ltd. (Mumbai, India) for the editing of the study.

\section{References}

1. Zhang Z, Pan J, Li L, Wang Z, Xiao W and Li N: Survey of risk factors contributed to lymphatic metastasis in patients with oral tongue cancer by immunohistochemistry. J Oral Pathol Med 40: 127-134, 2011.

2. Nagabhushan Kalburgi S, Khan NN and Gray SJ: Recent gene therapy advancements for neurological diseases. Discov Med 15: 111-119, 2013.

3. Barbosa M, Henrique M, Pinto-Basto J, Claes K and Soares G: Prostate cancer in Cowden syndrome: somatic loss and germline mutation of the PTEN gene. Cancer Genet 204: 224-225, 2011.

4. Feng ZZ, Chen JW, Yang ZR, Lu GZ and Cai ZG: Expression of PTTGI and PTEN in endometrial carcinoma: correlation with tumorigenesis and progression. Med Oncol 29: 304-310, 2012.

5. Huang SH and O'Sullivan B: Oral cancer: Current role of radiotherapy and chemotherapy. Med Oral Patol Oral Cir Bucal 18: e233-e240, 2013

6. Vasko V, Espinosa AV, Scouten W, et al: Gene expression and functional evidence of epithelial-to-mesenchymal transition in papillary thyroid carcinoma invasion. Proc Natl Acad Sci USA 104: 2803-2808, 2007.

7. Tang CH and Tsai CC: CCL2 increases MMP-9 expression and cell motility in human chondrosarcoma cells via the Ras/Raf/MEK/ERK/NF- $\mathrm{B}$ signaling pathway. Biochem Pharmacol 83: 335-344, 2012. 
8. Esteller M: Cancer epigenomics: DNA methylomes and histone-modification maps. Nat Rev Genet 8: 286-298, 2007.

9. Tshering Vogel DW, Zbaeren P and Thoeny HC: Cancer of the oral cavity and oropharynx. Cancer Imaging 10: 62-72, 2010.

10. Yip KW, Li A, Li JH, et al: Potential utility of BimS as a novel apoptotic therapeutic molecule. Mol Ther 10: 533-544, 2004.

11. Keniry $M$ and Parsons R: The role of PTEN signaling perturbations in cancer and in targeted therapy. Oncogene 27: 5477-5485, 2008

12. Wu B, Wang X, Chi ZF, et al: Ursolic acid-induced apoptosis in K562 cells involving upregulation of PTEN gene expression and inactivation of the PI3K/Akt pathway. Arch Pharm Res 35: 543-548, 2012

13. Gu T, Zhang Z, Wang J, Guo J, Shen WH and Yin Y: CREB is a novel nuclear target of PTEN phosphatase. Cancer Res 71: 2821-2825, 2011.

14. Rachmiel A, Aizenbud D and Peled M: Enhancement of bone formation by bone morphogenetic protein-2 during alveolar distraction: an experimental study in sheep. J Periodontol 75: 1524-1531, 2004.

15. Liu H, Liang SL, Kumar S, Weyman CM, Liu W and Zhou A Statins induce apoptosis in ovarian cancer cells through activation of JNK and enhancement of Bim expression. Cancer Chemother Pharmacol 63: 997-1005, 2009.
16. Ou Y, Ma L, Ma L, Huang Z, Zhou W, Zhao C, Zhang B, Song Y, Yu C and Zhan Q: Overexpression of cyclin B1 antagonizes chemotherapeutic-induced apoptosis through PTEN/Akt pathway in human esophageal squamous cell carcinoma cells. Cancer Biol Ther 14: 45-55, 2013.

17. Ma WJ,Lv GD, Tuersun A, et al: Role of microRNA-21 and effect on PTEN in Kazakh's esophageal squamous cell carcinoma. Mol Biol Rep 38: 3253-3260, 2011.

18. Iwatsuki M, Mimori K, Fukagawa T, et al: The clinical significance of vimentin-expressing gastric cancer cells in bone marrow. Ann Surg Oncol 17: 2526-2533, 2010.

19. Häyry V, Mäkinen LK, Atula T, et al: Bmi-1 expression predicts prognosis in squamous cell carcinoma of the tongue. Br J Cancer 102: 892-897, 2010.

20. Pickhard AC, Margraf J, Knopf A, Stark T, et al: Inhibition of radiation induced migration of human head and neck squamous cell carcinoma cells by blocking of EGF receptor pathways. BMC Cancer 11: 388, 2011.

21. Leslie NR, Yang X, Downes CP and Weijer CJ: PtdIns( $3,4,5)$ $\mathrm{P}(3)$-dependent and -independent roles for PTEN in the control of cell migration. Curr Biol 17: 115-125, 2007. 